\title{
Schedulability analysis of mixed-criticality systems with multiple frequency specifications
}

\author{
Sanjoy Baruah \\ Department of Computer Science \\ The University of North Carolina \\ Chapel Hill, NC. USA \\ baruah@cs.unc.edu
}

\begin{abstract}
In mixed-criticality systems functionalities of different criticalities, that need to have their correctness validated to different levels of assurance, co-exist upon a shared platform. Multiple specifications at differing levels of assurance may be provided for such systems; the specifications that are trusted at very high levels of assurance tend to be more conservative than those at lower levels of assurance. Prior research on the scheduling of such mixed-criticality systems has primarily focused upon the case where multiple estimates of the worst-case execution time (WCET) of pieces of code are provided; in this paper, a model is considered in which multiple estimates are instead provided for the rate at which event-triggered processes are executed. An algorithm is derived for scheduling such systems upon a preemptive uniprocessor; the effectiveness of this algorithm is demonstrated quantitatively via the speedup factor metric.
\end{abstract}

\section{Keywords}

HI and LO criticality; uniprocessor scheduling; Liu \& Layland tasks; multiple period estimations; speedup bound

\section{INTRODUCTION}

Recurrent task models are widely used to model eventtriggered phenomena that occur repeatedly: each event gives rise to a job that needs to be executed. If these triggering events occur arbitrarily frequently then it is not possible to offer deterministic guarantees as to when each job will receive execution. Often, however, lower bounds can be estimated beforehand on the minimum amount of time that must elapse between successive occurrences of these events, and performance guarantees made under the assumption that these bounds are correct. Some commonly-used recurrent task models include the Liu \& Layland (LL) model [10] and the 3-parameter sporadic tasks model [11]. In this paper, we will primarily focus upon the Liu \& Layland (henceforth called the LL) model. Each task $\tau_{i}$ in the LL model

Permission to make digital or hard copies of all or part of this work for personal or classroom use is granted without fee provided that copies are not made or distributed for profit or commercial advantage and that copies bear this notice and the full citation on the first page. Copyrights for components of this work owned by others than ACM must be honored. Abstracting with credit is permitted. To copy otherwise, or republish, to post on servers or to redistribute to lists, requires prior specific permission and/or a fee. Request permissions from permissions@ acm.org.

EMSOFT'16, October 01-07, 2016, Pittsburgh, PA, USA

(C) 2016 ACM. ISBN 978-1-4503-4485-2/16/10 . \$ $\$ 15.00$

DOI: http://dx.doi.org/10.1145/2968478.2968488 is characterized by two parameters: a worst-case execution time (WCET) $C_{i}$, and a minimum inter-arrival separation $T_{i}$ (often simply called the period of the task). Such a task generates a potentially infinite sequence of jobs at run-time, with successive job-arrivals assumed to be separated by at least $T_{i}$ time units and each job needing to execute for at most $C_{i}$ time units by a deadline that occurs $T_{i}$ time units after its arrival time.

Mixed-criticality scheduling theory arose out of a recognition of the reality that for many systems the true values of the parameters characterizing a task (i) are very difficult to determine precisely, and (ii) may be extremely conservative in the sense that they are far more pessimistic than the typical or average value. (For instance, advanced architectural features found in modern processors, such as memory hierarchies, deep pipelining, speculative out-of-order execution, etc., often result in the worst-case execution time of a task upon such a processor being multiple orders of magnitude greater than the execution time experienced by the overwhelmingly vast majority of jobs of the task.) Under mixed-criticality scheduling, one may choose to specify multiple estimates of a single parameter of a task, the different estimates being made at different levels of assurance. Each task is assigned a criticality, and the performance guarantee is that all the tasks will execute correctly if the least conservative parameter estimates are correct, while less critical tasks are not required to execute correctly if the less conservative parameter estimates turn out to be incorrect - see Example 1 for an illustration.

Much prior research on mixed-criticality scheduling (see, e.g., the survey [8] by Burns \& Davis) has focused upon models in which each task is characterized by multiple WCET parameters, the different parameters representing estimates, at different levels of assurance, of the actual WCET of the task. It has also been observed $[4,8]$ that it sometimes makes sense to specify multiple period parameters for tasks that are modeling event-triggered phenomena, since the precise pattern of triggering events is typically unknown beforehand and the period parameter denotes an estimate of the minimum duration between successive triggering events. A few papers $[1,7]$ have indeed considered this possibility; in this research we provide a comprehensive analysis of this aspect of mixed criticalities. That is, we primarily focus our attention on just the period parameter, and analyze situations in which multiple estimates are provided for the period parameter of each task, the different estimates being made as different levels of assurance. We illustrate via an example:

Example 1. Consider a system in which two indepen- 
dent pieces of event-triggered code are to be implemented upon a shared preemptive processor. The system developer models these pieces of code as LL tasks $\tau_{1}$ and $\tau_{2}$, and determines that these tasks have WCET parameters of two and five respectively (i.e., $C_{1}=2$ and $C_{2}=5$ ). The system developer further estimates that successive triggerings of $\tau_{1}$ will be at least twenty time units apart, and successive triggerings of $\tau_{2}$ will be at least six time units apart. From the system developer's perspective, therefore, $T_{1}=20$ and $T_{2}=6$ and this system thus has a utilization [10] of $\frac{2}{20}+\frac{5}{6} \approx 0.93$. The system developer consequently concludes, by the well-known utilization-based Earliest Deadline First (EDF) schedulability test [10] that the system would meet all deadlines if scheduled using EDF.

Now, let us suppose that task $\tau_{1}$ is responsible for some highly safety-critical function, and is therefore subject to certification by a Certification Authority (CA) - $\tau_{2}$ is not subject to such certification. But the CA makes more conservative assumptions than the system developer does and assumes that successive events triggering $\tau_{1}$ may occur as frequently as ten time units apart. ${ }^{1}$ The CA hence requires that the safety-critical task $\tau_{1}$ be modeled as having a period of 10 , rather than 20 . The utilization of the system, from the CA's perspective, is $\frac{2}{10}+\frac{5}{6} \approx 1.03$, and EDF cannot guarantee to meet all deadlines (indeed, since the utilization is $>1$ no algorithm can guarantee to do so). An EDF-scheduled implementation will therefore not pass certification by the CA.

An alternative implementation would assign higher scheduling priority to $\tau_{1}$ — this would pass certification since each job of $\tau_{1}$ is now guaranteed execution immediately upon arrival. But consider a situation in which one job of each task arrives simultaneously: $\tau_{1}$ 's job would execute first for two time units, thereby denying $\tau_{2}$ 's job an opportunity to execute for five time units by its deadline regardless of when the next job of $\tau_{1}$ may arrive. Thus, although this system design is accepted by the CA, deadlines are missed even when the system behaves as expected by the system developer (i.e., as a system with effective utilization $\approx 0.93$ ). A preferred scheduling policy would be one that has all the jobs meet their deadlines if the system developer's assumptions are correct, and all jobs of $\tau_{1}$ meet their deadlines (while those of $\tau_{2}$ need not) if the system developer's assumptions are incorrect but the CA's assumptions are correct. We will derive such a scheduling policy in this paper.

(We point out here that in order to pass certification, the system developer must ensure that each of $\tau_{1}$ 's jobs completes within a deadline that is 10 time units after its arrival (since the jobs are assumed to have deadlines after a duration of one period after their arrival time), even if the jobs do arrive 20 time units apart. Hence from the system developer's perspective the task is better modeled as a 3parameter sporadic task [11] — this task model is described in Section 2.1 - with WCET 2, relative deadline 10, and period 20.)

\footnotetext{
${ }^{1}$ The CA may also make a more conservative estimate of the WCET $C_{1}$ than the system developer does; we will assume in this paper that both the system developer and the CA make the same estimate of all WCETs, thus allowing us to focus on variations in the period estimates. Sec. 6 discusses extensions that enable the concurrent consideration of uncertainties along both the WCET and period dimensions.
}

This research. Most prior research (see [8] for a survey) on mixed-criticality scheduling has looked at systems specified with multiple WCET parameters. The research described in this document is, by contrast, focused on dealing with pessimism in the period perimeter; this work thus complements the large body of prior work dealing with pessimism along the WCET dimension. For MC systems in which certification pessimism is expressed along the period dimension we present, prove the correctness of, and provide a quantitative metric of efficacy for, an algorithm for scheduling such systems upon a preemptive uniprocessor platform. As is fairly routine in the mixed-criticality scheduling theory literature, we primarily restrict our attention here to systems in which two criticality levels, designated LO and HI, are defined; we briefly address, in Section 6, possible extension to more levels.

Related work. We will not review here the vast body of research that exists concerning the mixed-criticality scheduling of task systems with multiple WCET specifications see [8] for a comprehensive survey. Preemptive uniprocessor EDF-based scheduling of task systems specified with pessimism expressed along the frequency dimension -the subject of this paper- was first studied in [1], and subsequently extended to fixed-priority scheduling in [5]. Burns and Davis [7] considered the scheduling of streams of periodic real-time traffic on controller-area networks (CANs), with the traffic streams being specified by multiple period parameters at different levels of pessimism.

The research described in this manuscript may be considered to be an extension (and improvement) of the results reported in [1]. The scheduling algorithm that was presented in [1] was proved correct there, but no quantitative metrics of its efficacy were derived. In this paper, we derive and prove correct a scheduling algorithm that strictly dominates the one in [1] - this is formally shown in Section 5.2. We have additionally derived, in Sections 5.3-5.4, a quantitative metric of its effectiveness by showing that this algorithm has a speedup bound of $(1+\sqrt{3} / 2)$ or $\approx 1.866$.

Organization. The mixed-criticality model studied in this paper is formally defined in Section 2; some additional definitions that will prove useful later in this paper are also presented here. The main technical contribution in this paper - an algorithm for scheduling mixed-criticality task systems upon preemptive uniprocessor platforms - is presented in Section 3; a proof of its correctness is derived in Section 4. Section 5 characterizes the effectiveness of this algorithm by both showing that it strictly dominates the prior state of the art, and by deriving a speedup bound for it.

\section{MODEL \& DEFINITIONS}

We start out formally defining the task model that we will be using. We characterize a mixed-criticality Liu $\&$ Layland (MC LL) task by a 4-tuple of parameters:

$$
\tau_{i}=\left(\chi_{i}, C_{i}, T_{i}(\mathrm{HI}), T_{i}(\mathrm{LO})\right),
$$

where

- $\chi_{i} \in\{\mathrm{LO}, \mathrm{HI}\}$ denotes the criticality of the task (with HI being "more" critical than LO);

- $C_{i}$ denotes the WCET of the task; 
- $T_{i}(\mathrm{HI})$ denotes a conservative estimate of the minimum amount of time that will elapse between the arrival times of successive jobs of $\tau_{i}$; and

- $T_{i}(\mathrm{LO})$ denotes a less conservative (equivalently, more optimistic) estimate of the minimum amount of time that will elapse between the arrival times of successive jobs of $\tau_{i}$.

We make the assumption that $T_{i}(\mathrm{HI}) \leq T_{i}$ (LO) for all tasks $\tau_{i}$. We also assume that for each LO-criticality task $\tau_{i}$, $T_{i}(\mathrm{HI})=T_{i}$ (LO); i.e., we will not admit jobs of such tasks more frequently than in accordance with their LO-criticality periods.

A MC LL task system $\tau$ is a finite collection of independent MC tasks: $\tau=\left\{\tau_{1}, \tau_{2}, \ldots, \tau_{n}\right\}$. We study the problem of scheduling such MC LL task systems on a fully preemptive uniprocessor.

In this notation, the task system of Example 1 is specified as follows:

$$
\tau=\left\{\tau_{1}=(\mathrm{HI}, 2,10,20), \tau_{2}=(\mathrm{LO}, 5,6,6)\right\}
$$

Behaviors. Any execution of the system satisfying the property that for each task $\tau_{i}$, successive jobs of $\tau_{i}$ arrive at least $T_{i}$ (LO) time units apart, is said to exhibit a LOcriticality behavior. Any execution of the system that does not exhibit LO-criticality behavior but which satisfies the property that for each task $\tau_{i}$, successive jobs of $\tau_{i}$ arrive at least $T_{i}(\mathrm{HI})$ time units apart is said to exhibit a HI-criticality behavior. Any execution that does not exhibit HI-criticality or LO-criticality behavior is said to be erroneous.

Correctness. An algorithm for scheduling MC LL task systems is said to be correct if and only if it satisfies the property that

- Each job of each task $\tau_{i}$ completes execution within $T_{i}(\mathrm{HI})$ units of arrival, in all LO-criticality behaviors of the system ${ }^{2}$; and

- Each job of each HI-criticality task $\tau_{i}$ (i.e., all $\tau_{i}$ with $\left.\chi_{i}=\mathrm{HI}\right)$ completes execution within $T_{i}(\mathrm{HI})$ units of arrival, in all HI-criticality behaviors of the system.

\subsection{The 3-parameter sporadic task model}

The correctness requirement of MC LL tasks in LO-criticality behaviors is conveniently expressed using the 3-parameter sporadic task model [11], which generalizes the Liu \& Layland (LL) model. A 3-parameter sporadic task $\tau_{i}=\left(C_{i}, D_{i}, T_{i}\right)$ is characterized by three parameters: a WCET $C_{i}$ and a pe$\operatorname{riod} T_{i}$ as in the LL model, plus an additional relative deadline parameter $D_{i}$. Such a task generates an unbounded sequence of jobs, with each job having an execution requirement $\leq C_{i}$ and successive arrivals at least $T_{i}$ time units apart. However, each job is required to complete by a deadline that is $D_{i}$ (rather than $T_{i}$, as in the LL model) time units after its arrival time.

The EDF-schedulability-analysis of systems of 3-parameter sporadic tasks on preemptive uniprocessors has been extensively studied (see, e.g. [10,6]); such study has yielded exact

\footnotetext{
${ }^{2}$ Note that the deadlines for the jobs are $T_{i}(\mathrm{HI})$ time-units after arrival and not $T_{i}(\mathrm{LO})$; this is a consequence of the fact that jobs of LL tasks are expected to complete execution within one period of their arrival and the period, conservatively estimated, is $T_{i}(\mathrm{HI})$. (For LO-criticality tasks $\tau_{i}$ we have $T_{i}(\mathrm{LO})=T_{i}(\mathrm{HI})$.)
}

preemptive uniprocessor EDF-schedulability analysis algorithms for 3-parameter sporadic task systems. These algorithms make use of the concept of the demand bound function [6] of 3-parameter sporadic tasks; the demand bound function $\operatorname{DBF}\left(\tau_{i}, t\right)$ of 3 -parameter sporadic task $\tau_{i}$ for an interval-length $t$ is defined as follows:

$$
\operatorname{DBF}\left(\tau_{i}, t\right) \stackrel{\text { def }}{=} \max \left(0,\left(\left\lfloor\frac{t-D_{i}}{T_{i}}\right\rfloor+1\right) \times C_{i}\right)
$$

The load of a 3-parameter sporadic task system $\tau$ is defined as follows:

$$
\operatorname{LOAD}(\tau) \stackrel{\text { def }}{=} \max _{t \geq 0} \frac{\sum_{\tau_{i} \in \tau} \operatorname{DBF}\left(\tau_{i}, t\right)}{t}
$$

It has been shown [6] that a necessary and sufficient condition for 3-parameter sporadic task system $\tau$ to be EDFschedulable on a unit-speed preemptive uniprocessor is that

$$
\operatorname{LOAD}(\tau) \leq 1
$$

Pseudo-polynomial algorithms are known $[6,13,14]$ for computing $\operatorname{LOAD}(\tau)$, for task systems $\tau$ possessing the additional property that the quantity $\left(\sum_{\tau_{i} \in \tau} C_{i} / T_{i}\right)$ is bounded from above by a constant $<1$. Polynomial-time approximation schemes (PTAS's) have also been derived that are able to compute an approximation to $\operatorname{LOAD}(\tau)$ in polynomial time, to any desired degree of accuracy [9].

The following definition specifies a transformation from a mixed-criticality LL task system to a 3-parameter sporadic task system; we will be using this transformation in later sections of this paper.

DeFINITION 1. Let $x$ denote a positive real number, $0<$ $x \leq 1$. For any mixed-criticality $L L$ task $\tau_{i}$, we can define a corresponding 3-parameter sporadic task $\hat{\tau}_{i}(x)$ as follows :

$$
\begin{aligned}
\hat{\tau}_{i}(x)=\left(\hat{C}_{i}, \hat{D}_{i}, \hat{T}_{i}\right) \stackrel{\text { def }}{=} \\
\begin{cases}\left(C_{i}, T_{i}(\mathrm{HI}), T_{i}(\mathrm{LO})\right), & \text { if } \chi_{i}=\mathrm{LO} \\
\left(C_{i}, x T_{i}(\mathrm{HI}), T_{i}(\mathrm{LO})\right), & \text { if } \chi_{i}=\mathrm{HI}\end{cases}
\end{aligned}
$$

For MC LL task system $\tau$, let $\hat{\tau}(x)$ denote the 3-parameter sporadic task system obtained from $\tau$ by applying the $\hat{\tau}_{i}(x)$ transformation to each task in $\tau$ :

$$
\hat{\tau}(x) \stackrel{\text { def }}{=}\left\{\hat{\tau}_{1}(x), \hat{\tau}_{2}(x), \ldots, \hat{\tau}_{n}(x)\right\}
$$

In words, $\hat{\tau}_{i}(x)=\left(\hat{C}_{i}, \hat{D}_{i}, \hat{T}_{i}\right)$ is a 3 -parameter sporadic task that has the same WCET as $\tau_{i}\left(\hat{C}_{i}=C_{i}\right)$, and the same period as the LO-criticality period of $\tau_{i}\left(\hat{T}_{i}=T_{i}(\mathrm{LO})\right)$. If $\tau_{i}$ is of LO criticality, then $\hat{\tau}_{i}$ 's relative deadline is equal to its period $\left(\hat{D}_{i}=T_{i}(\mathrm{HI})\right.$, which equals $\left.T_{i}(\mathrm{LO})\right)$. However, if $\tau_{i}$ is a HI-criticality task, then $\hat{\tau}_{i}$ is assigned a relative deadline parameter that is the HI-criticality period parameter of $\tau_{i}$ scaled by the factor $x$ (i.e., $\hat{D}_{i}=x T_{i}(\mathrm{HI}) \leq T_{i}(\mathrm{HI})$ ).

In this notation, letting $\tau$ denote the task system in Expression (1), we would have

$$
\begin{aligned}
\hat{\tau}(1) & =\left\{\hat{\tau}_{1}(1)=(\mathrm{HI}, 2,10,20), \hat{\tau}_{2}(1)=(\mathrm{LO}, 5,6,6)\right\} \\
\hat{\tau}(0.5) & =\left\{\hat{\tau}_{1}(0.5)=(\mathrm{HI}, 2,5,20), \hat{\tau}_{2}(0.5)=(\mathrm{LO}, 5,6,6)\right\}
\end{aligned}
$$

Using this notation, the correctness requirement for any mixed-criticality LL task $\tau_{i}$ in a LO-criticality behavior may be modeled by the 3 -parameter sporadic task $\hat{\tau}_{i}(1)$. 


\section{THE ALGORITHM}

Let $\tau=\left\{\tau_{1}, \tau_{2}, \ldots, \tau_{n}\right\}$ denote the MC LL task system that is to be scheduled on a unit-speed preemptive processor. Suppose that at some time-instant $t^{*}$ a job of some task $\tau_{j}$ arrives less than $T_{j}(\mathrm{LO})$ time units after the arrival of the previous job of $\tau_{j}$. The behavior is no longer compliant with LO-criticality specifications, and we assert that a criticality level change has occurred at time-instant $t^{*}$. Henceforth we are not required to demonstrate correct execution of LOcriticality tasks and can discard all LO-criticality jobs. Informally, we would like to ensure for each HI-criticality task $\tau_{i}$ that is active - i.e., has arrived but has not completed execution - at time $t^{*}$ that there is enough capacity available on the processor to ensure that $\tau_{i}$ 's currently-active job completes execution by its deadline, provided only HI-criticality jobs execute henceforth. To achieve this, we will ensure that as long as the system is behaving according to its LOcriticality specifications, $\tau_{i}$ 's job would have completed well before its actual deadline. We will thus ensure that if it is active at time-instant $t^{*}$ there is sufficient computing capacity freed up by the discarded LO-criticality tasks to schedule $\tau_{i}$ 's job to completion by its actual deadline.

Our scheduling algorithm for achieving this can be thought of operating in three phases. The first phase is occurs prior to run-time: a schedulability test is applied to the system and if successful, a scaling parameter $x$ is computed. This readies the system for the second, run-time phase of the algorithm. During this second phase, jobs are initially dispatched under the assumption that the system will behave according to the LO-criticality specifications. If this is violated, a criticality level change occurs and the algorithm enters a third phase.

\subsection{The pre-runtime phase}

We seek to determine, prior to run-time, whether a given mixed-criticality LL task system $\tau$ can be successfully scheduled by our algorithm; if so, we compute a scaling parameter $x$ by which we will initially shrink the deadlines of all HI-criticality jobs in order to ensure that they all complete well before their actual deadlines.

We first introduce some notation:

- The term $U_{\mathrm{LO}}^{\mathrm{LO}}\left(U_{\mathrm{HI}}^{\mathrm{HI}}\right.$, respectively) denotes the cumulative utilization of the LO-criticality (HI-criticality, resp.) tasks assuming the LO-criticality (HI-criticality, resp.) period estimates:

$$
\begin{aligned}
& U_{\mathrm{LO}}^{\mathrm{LO}} \stackrel{\text { def }}{=} \sum_{\tau_{i} \in \tau \wedge \chi_{i}=\mathrm{LO}} \frac{C_{i}}{T_{i}(\mathrm{LO})} \\
& U_{\mathrm{HI}}^{\mathrm{HI}} \stackrel{\text { def }}{=} \sum_{\tau_{i} \in \tau \wedge \chi_{i}=\mathrm{HI}} \frac{C_{i}}{T_{i}(\mathrm{HI})}
\end{aligned}
$$

- The term $\ell_{\mathrm{LO}}$ denotes the load of the system (see Expression (3)) assuming that the LO-criticality period estimates are correct, and each HI-criticality job must complete within an interval of duration equal to the HIcriticality period estimate of the task that generated it:

$$
\ell_{\mathrm{LO}} \stackrel{\text { def }}{=} \operatorname{LOAD}(\hat{\tau}(1))
$$

(Recall that, as stated in both Footnote 2 and after Definition 1, the correctness requirement for any mixed-criticality LL task $\tau_{i}$ in a LO-criticality behavior is modeled as the 3 -parameter sporadic task $\hat{\tau}_{i}(1)$. Hence $\ell_{\mathrm{LO}}$ represents the maximum load that may be generated by $\tau$ in any LO-criticality behavior.)

The pre-processing algorithm is depicted in pseudo-code form in Algorithm 1; in Section 4 we will show that if Algorithm 1 returns success for a given task system $\tau$, then $\tau$ is scheduled correctly by the run-time dispatcher described in Section 3.2 below. As can be seen from the pseudo-code in Algorithm 1, $U_{\mathrm{LO}}^{\mathrm{LO}}$ and $U_{\mathrm{HI}}^{\mathrm{HI}}$ are first computed; if they sum to at most one (this is tested in Line $\mathrm{C} 1$ ), then $x$ is assigned the value one. Else, $\ell_{\mathrm{LO}}$ is computed and an additional condition checked (Line C2); if this condition evaluates to true, then $x$ is assigned a value specified in Line L2. If the condition in Line C2 evaluates to false, a form of search is conducted for a suitable value of $x$ - the test of Line C3 determines whether this search has yielded success (line L4) or failure (Line L5). This pre-processing phase is deemed successful if and only if the pre-processing algorithm returns success, in which case the computed value of $x$ is used as the scaling factor for HI-criticality deadlines in the subsequent, run-time phase.

\subsection{Run-time dispatching}

Dispatching of jobs during run-time occurs according to the following rules:

1. There is a criticality level indicator $\Gamma$, initialized to LO.

2. While $(\Gamma \equiv \mathrm{LO})$, (this corresponds to the second phase of our algorithm as described in the overview.)

(a) If a job of task $\tau_{i} \in \tau$ arrives at time $t$, it is assigned a scheduling deadline as follows

$$
\begin{cases}t+T_{i}(\mathrm{LO}) & \text { if } \chi_{i}=\mathrm{LO} \\ t+x \times T_{i}(\mathrm{HI}) & \text { if } \chi_{i}=\mathrm{HI}\end{cases}
$$

That is, each LO-criticality job is assigned a deadline equal to its arrival time plus its period, while each HI-criticality job is assigned a deadline equal to its arrival time plus its HI-criticality period scaled by the scaling factor $x$.

(b) At each instant the earliest-deadline waiting job is selected for execution (ties broken arbitrarily).

(c) If a job of some task $\tau_{i}$ arrives earlier than $T_{i}(\mathrm{LO})$ time units after the arrival of the previous job of the same task, then $\Gamma \leftarrow$ HI.

3. Once $(\Gamma \equiv \mathrm{HI})$ - this corresponds to the third phase of our algorithm, which may or may not execute during any given run of the system

(a) All currently-active LO-criticality jobs are immediately discarded. The deadline of each currently active HI-criticality job is changed to become equal to its arrival time plus the HI-criticality period (the $T_{i}(\mathrm{HI})$ ) of the task that generated it). That is, if a job of $\tau_{i}$ that was released at some time $t$ is active, its deadline, for scheduling purposes, is henceforth $t+T_{i}(\mathrm{HI})$.

(b) If a job of $\tau_{i}$ arrives at some time $t$, it is assigned a deadline equal to $t+T_{i}(\mathrm{HI})$ if $\chi_{i}=\mathrm{HI}$, and discarded if $\chi_{i}=$ LO. 


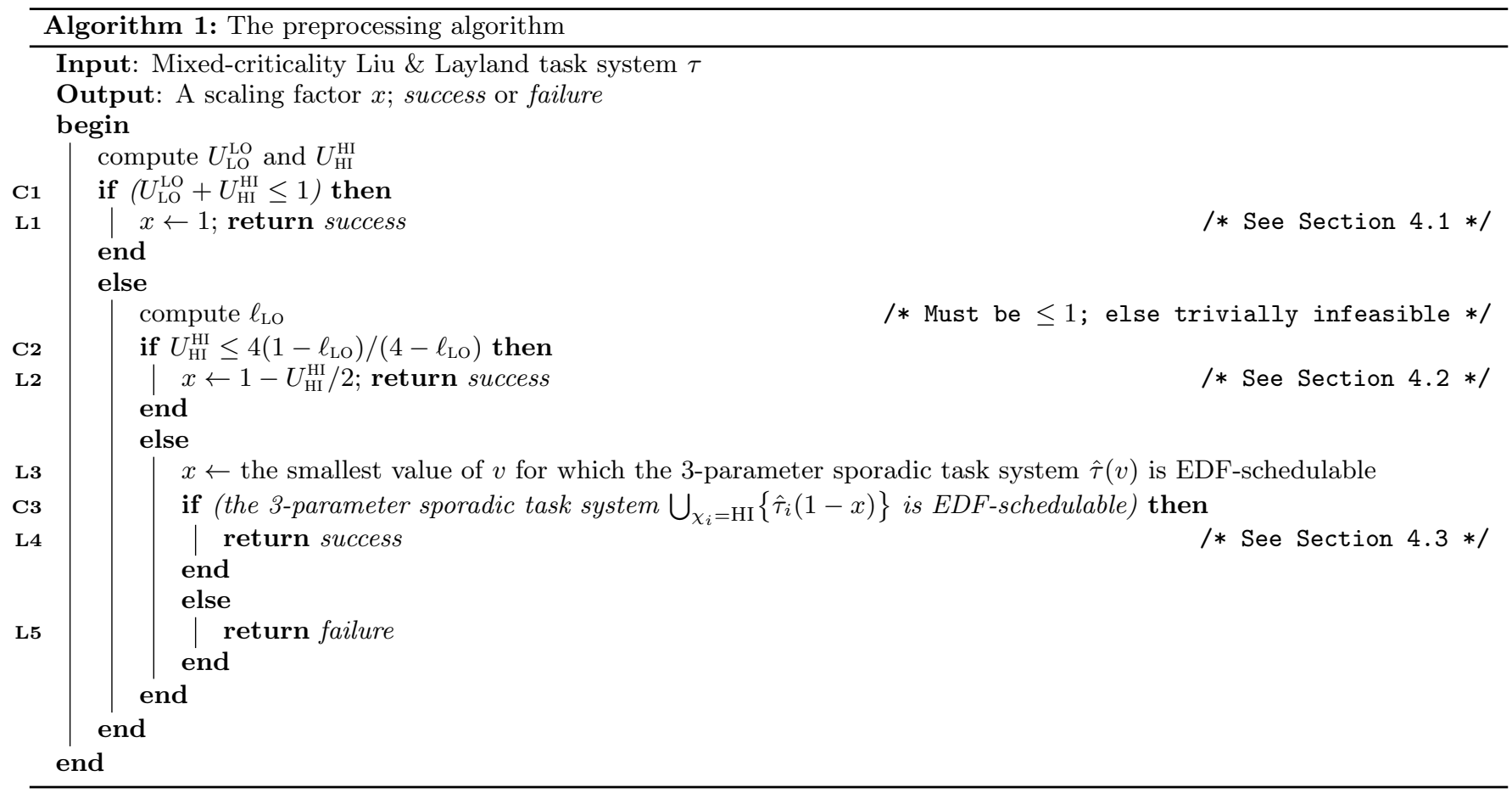

(c) At each instant the earliest-deadline waiting job is selected for execution (ties broken arbitrarily).

4. An additional rule could specify the circumstances when $\Gamma$ gets reset to LO. This could happen, for instance, if no HI-criticality jobs are active at some instant in time. (We will not discuss the process of resetting $\Gamma \leftarrow$ LO any further in this document.)

\section{PROOF OF CORRECTNESS}

We now show that if Algorithm 1 declares success for a given task system $\tau$, then $\tau$ is scheduled correctly by the runtime dispatcher of Section 3.2. Observe that Algorithm 1 may declare success at three places: Lines L1, L2, and L4; we consider each case separately, in Sections 4.1-4.3.

\subsection{Returns success at Line $\mathrm{L1}$}

This is the simplest case; informally, it corresponds to the situation where the task system is successfully scheduled using an isolation-based approach. (That is, the regular (i.e., non-MC) LL task system obtained when each task is modeled as a regular LL task with period equal to its period estimate at its own criticality level is determined to be schedulable using EDF.)

LEMMA 1. If the algorithm returns success at Line L1 for a given task system $\tau$, then $\tau$ is scheduled correctly by the run-time dispatcher described in Section 3.2.

Proof: By setting the scaling factor $x$ equal to one, we are in effect over-approximating the computational demand of each HI-criticality task $\tau_{i}=\left(\mathrm{HI}, C_{i}, T_{i}(\mathrm{HI}), T_{i}(\mathrm{LO})\right)$ as a regular LL task with WCET $C_{i}$ and period $T_{i}(\mathrm{HI})$, and representing each LO-criticality task $\tau_{i}=\left(\mathrm{HI}, C_{i}, T_{i}(\mathrm{HI}), T_{i}(\mathrm{LO})\right)$ as a regular LL task with WCET $C_{i}$ and period $T_{i}(\mathrm{LO})$. The utilization of the resulting regular LL task system is

$$
\begin{aligned}
& \sum_{i: \chi_{i}=\mathrm{LO}} \frac{C_{i}}{T_{i}(\mathrm{LO})}+\sum_{i: \chi_{i}=\mathrm{HI}} \frac{C_{i}}{T_{i}(\mathrm{HI})} \\
& \quad=\left(U_{\mathrm{LO}}^{\mathrm{LO}}+U_{\mathrm{HI}}^{\mathrm{HI}}\right) \leq 1
\end{aligned}
$$

This task system is therefore schedulable by EDF to always meet all deadlines. It follows that all deadlines of all tasks are met under both LO-criticality and HI-criticality behaviors when scheduled using EDF.

\subsection{Returns success at Line L2}

This case turns out to be the most challenging to analyze. We proceed in two steps. The first step - proving that no deadlines are missed in any LO-criticality behavior - is fairly straightforward; this is shown in Lemma 2. We then adapt and apply some recently-introduced techniques and ideas from [3] to prove, in Lemma 3, that no deadlines are missed in any HI-criticality behavior as well.

FACT 1. If the conditional expression of Line C2 evaluates to true, then

$$
U_{\mathrm{HI}}^{\mathrm{HI}} \leq 2\left(1-\ell_{\mathrm{LO}}\right)
$$

Proof: Suppose that the conditional expression of Line C2 evaluates to true; i.e., we have

$$
U_{\mathrm{HI}}^{\mathrm{HI}} \leq \frac{4\left(1-\ell_{\mathrm{LO}}\right)}{4-\ell_{\mathrm{LO}}}
$$

We will show that Inequality 7 must also hold by showing below that

$$
\frac{4\left(1-\ell_{\mathrm{LO}}\right)}{4-\ell_{\mathrm{LO}}} \leq 2\left(1-\ell_{\mathrm{LO}}\right)
$$


Observe that

$$
\begin{aligned}
& 2\left(1-\ell_{\mathrm{LO}}\right)-\frac{4\left(1-\ell_{\mathrm{LO}}\right)}{4-\ell_{\mathrm{LO}}} \\
= & 2\left(1-\ell_{\mathrm{LO}}\right)\left(1-\frac{2}{4-\ell_{\mathrm{LO}}}\right) \\
= & 2\left(1-\ell_{\mathrm{LO}}\right)\left(\frac{2-\ell_{\mathrm{LO}}}{4-\ell_{\mathrm{LO}}}\right)
\end{aligned}
$$

which is $\geq 0$ for all $\ell_{\mathrm{LO}} \in[0,1]$. We have thus shown that

$$
2\left(1-\ell_{\mathrm{LO}}\right)-\frac{4\left(1-\ell_{\mathrm{LO}}\right)}{4-\ell_{\mathrm{LO}}} \geq 0
$$

from which it follows that

$$
2\left(1-\ell_{\mathrm{LO}}\right) \geq \frac{4\left(1-\ell_{\mathrm{LO}}\right)}{4-\ell_{\mathrm{LO}}}
$$

LEMma 2. If the algorithm returns success at Line L2, then no deadline is missed by the run-time dispatcher described in Section 3.2 upon any LO-criticality behavior.

Proof: We saw in Fact 1 above that $U_{\mathrm{HI}}^{\mathrm{HI}} \leq 2\left(1-\ell_{\mathrm{LO}}\right)$ if Line L2 is executed; since $x$ gets assigned the value $\left(1-\left(U_{\mathrm{HI}}^{\mathrm{HI}} / 2\right)\right)$ in Line L2, this value of $x$ is guaranteed to be $\geq \ell_{\mathrm{LO}}$.

Consider now any LO-criticality behavior of $\tau$. For any time interval $\left[t_{1}, t_{2}\right]$, let $\Delta\left(t_{1}, t_{2}\right)\left(\Delta^{\prime}\left(t_{1}, t_{2}\right)\right.$, respectively) denote the sum of the WCETs of jobs that have both arrival times and deadlines (scaled deadlines, respectively ${ }^{3}$ ) within this interval in this behavior. Consider a particular timeinterval $\left[t_{o}, t_{o}+t\right]$. Let $J_{k}$ denote any job that contributes to $\Delta^{\prime}\left(t_{o}, t_{o}+t\right)$, and let $a_{k}, d_{k}$ denote the arrival time and relative deadline (prior to scaling) of $J_{k}$. We have

$$
\begin{aligned}
a_{k} & +x d_{k} \leq t_{o}+t \\
& \Leftrightarrow \quad x d_{k} \leq t-\left(a_{k}-t_{o}\right) \\
& \Leftrightarrow \quad d_{k} \leq \frac{t}{x}-\frac{\left(a_{k}-t_{o}\right)}{x} \\
& \Rightarrow \quad d_{k} \leq \frac{t}{x}-\left(a_{k}-t_{o}\right) \quad(\text { Since } x \leq 1) \\
& \Rightarrow \quad a_{k}+d_{k} \leq \frac{t}{x}+t_{o}
\end{aligned}
$$

That is, any job contributing to $\Delta^{\prime}\left(t_{o}, t_{o}+t\right)$ also contributes to $\Delta\left(t_{o}, t_{o}+\frac{t}{x}\right)$; we therefore have

$$
\begin{aligned}
\Delta^{\prime}\left(t_{o}, t_{o}+t\right) & =\Delta\left(t_{o}, t_{o}+\frac{t}{x}\right) \\
& \leq \frac{t}{x} \times \ell_{\mathrm{LO}} \\
& \left.\leq t \text { (Since } x \geq \ell_{\mathrm{LO}}\right)
\end{aligned}
$$

We have thus shown that for any time interval $\left[t_{o}, t_{o}+t\right)$, the demand $\Delta^{\prime}\left(t_{o}, t_{o}+t\right)$ is no larger than the duration of the interval. Since the run-time dispatcher described in Section 3.2 effectively schedules any LO-criticality behavior using EDF with the scaled deadlines, it follows from the optimality of EDF that no deadlines will be missed in any LO-criticality behavior.

By Lemma 2 above, no deadlines are missed in any LOcriticality behavior. Let us now derive conditions that must

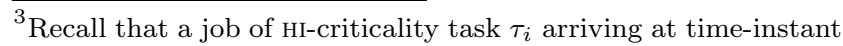
$t_{o}$ has its deadline at $\left(t_{o}+T_{i}\right)$ and scaled deadline at $\left(t_{o}+x T_{i}\right)$.
}

necessarily be satisfied by the task system $\tau$ in order for a deadline miss to occur in some HI-criticality behavior.

Let $I$ denote a minimal ${ }^{4}$ collection of jobs generated by $\tau$ for which a deadline miss occurs, and let $t_{f}$ denote the earliest instant at which this happens - see Figure 1. It follows from Lemma 2 that the algorithm has set the criticality level indicator $\Gamma$ to HI prior to time-instant $t_{f}$, and that the job to miss its deadline at $t_{f}$ is a HI-criticality job. Let us denote the time-instant at which $\Gamma$ was first assigned the value $\mathrm{HI}$ as $t^{*}$.

FACT 2. All jobs receiving any execution in the interval $\left[t^{*}, t_{f}\right)$ must have (absolute) deadlines no larger than $t_{f}$.

Proof: Suppose there is a job that has deadline larger than $t_{f}$ and receives some execution between $t^{*}$ and $t_{f}$, say in the interval $\left[t_{1}, t_{2}\right)$. This means that during $\left[t_{1}, t_{2}\right)$ there are no active jobs awaiting execution that have deadline at most $t_{f}$. Hence the collection of jobs obtained by considering only jobs with arrival time at least $t_{2}$ will also miss a deadline at $t_{f}$, which contradicts the assumed minimality of $I$.

Let $S^{*}$ denote the collection of jobs in $I$ that receive execution after $t^{*}$. Let $J_{0}$ denote the job that has the earliest arrival time in $S^{*}$. Let $a_{0}$ and $d_{0}$ denote its arrival time and absolute deadline, respectively; let $\tilde{d}_{0} \stackrel{\text { def }}{=} a_{0}+x\left(d_{0}-a_{0}\right)$ denote its scaled deadline.

FACT 3. Any LO-criticality job in I must have its absolute deadline $\leq a_{0}+x\left(t_{f}-a_{0}\right)$.

Proof: Note that no LO-criticality job with absolute deadline $>\tilde{d}_{0}$ will execute at or after $a_{0}$ - since such a job will only receive execution time before $t^{*}$, this would imply that $J_{0}$ has completed execution by time-instant $t^{*}$, hereby contradicting the definition of $J_{0}$.

Suppose there is a LO-criticality job with absolute deadline $>\tilde{d}_{0}$ that executes prior to time-instant $a_{0}$; consider the latest time-instant during which it executes. It must be the case that there are no active jobs with deadline at most $\tilde{d}_{0}$ awaiting execution at this time-instant, and the job sequence obtained by only considering jobs released after this timeinstant also misses a deadline. That contradicts the assumed minimality of $I$. Therefore any LO-criticality job in $I$ must have an absolute deadline $\leq \tilde{d}_{0}=a_{0}+x\left(d_{0}-a_{0}\right) \leq a_{0}+$ $x\left(t_{f}-a_{0}\right)$, applying the result of Fact 2 to conclude that $d_{0} \leq t_{f}$.

FACT 4. Any HI-criticality job in I that only receives execution prior to time-instant $t^{*}$ must have its absolute scaled deadline $\leq a_{0}+x\left(t_{f}-a_{0}\right)$.

Proof: If a HI-criticality job $J_{k} \in I$ with arrival time $a_{k}$, absolute deadline $d_{k}$, and scaled absolute deadline $\tilde{d}_{k}$ does not receive execution after $t^{*}$, there are two possibilities:

1. $a_{k} \geq a_{0}$. In this case $\tilde{d}_{k} \leq \tilde{d}_{0}$, since otherwise $J_{k}$ would not receive any execution after $a_{0}$, implying that it does not receive any execution before $t^{*}$, which

\footnotetext{
${ }^{4}$ By minimal, we mean that some deadline is missed when $I$ is scheduled, but no deadline miss would occur if any proper subset of $I$ were being scheduled.
} 
$S^{*}$ : jobs executing over $\left[t^{*}, t_{f}\right)$

$S_{1}$ : LO-criticality jobs executing prior to $t^{*}$

$S_{2}$ : HI-criticality jobs executing prior to $t^{*}$.

$C^{*}, C_{1}, C_{2}$ : cumulative execution requirements of jobs in $S^{*}, S_{1}, S_{2}$.

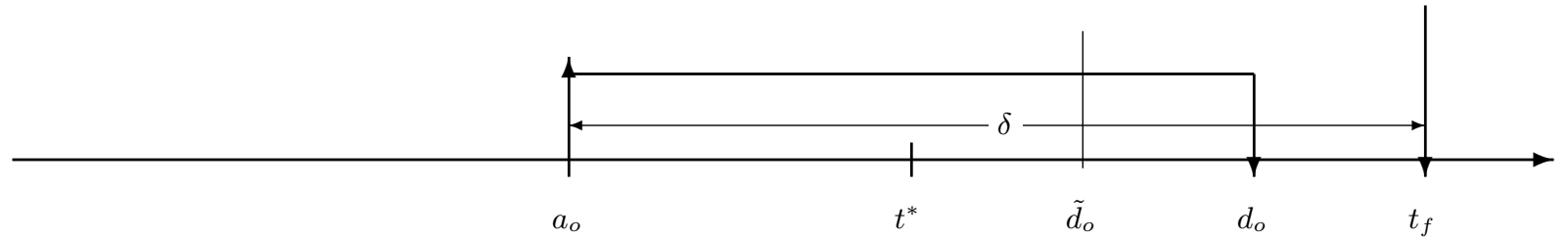

Figure 1: Notation. $S^{*}$ : jobs executing over $\left[t^{*}, t_{f}\right) . \quad S_{1}$ : LO-criticality jobs executing prior to $t^{*}$. $S_{2}$ : HIcriticality jobs executing prior to $t^{*}$.

combined with $J_{k}$ not receiving any execution after $t^{*}$, would mean that that the instance $I$ without $J_{k}$ would also see a deadline miss of $J$ at $t_{f}$ (thus contradicting the assumed minimality of $I$ ).

2. $a_{k}<a_{0}$. If $d_{k} \leq t_{f}$, then $\tilde{d}_{k}=a_{k}+x\left(d_{k}-a_{k}\right) \leq$ $a_{0}+x\left(t_{f}-a_{0}\right)$. Otherwise, if $d_{k}>t_{f}$, then again $\tilde{d}_{k} \leq \tilde{d}_{0}$ must hold, since otherwise we can remove $J_{k}$ from $I$ without affecting the deadline miss of $J$, thus contradicting the minimality of $I$.

The proof is completed by noting that $\tilde{d}_{k} \leq \tilde{d}_{0}=a_{0}+x\left(d_{0}-\right.$ $\left.a_{0}\right) \leq a_{0}+x\left(t_{f}-a_{0}\right)$.

Observe that $a_{k}+x d_{k}=\tilde{d}_{k} \leq a_{0}+x\left(t_{f}-a_{0}\right)$ implies that $d_{k} \leq a_{0} / x+\left(t_{f}-a_{0}\right)$ - this may be formally shown in a manner analogous to the derivation in the proof of Lemma 2 .

Let us define $S_{1}$ to be the collection of LO-criticality jobs of $I$, and $S_{2}$ to be the collection of HI-criticality jobs of $I$ that only receive execution before $t^{*}$. Therefore, the collection of jobs $I$ is the disjoint union of $S_{1}, S_{2}$ and $S^{*}$.

Let $C_{1}, C_{2}$, and $C^{*}$ denote respectively the sum of execution requirements of all the jobs in $S_{1} S_{2}$, and $S^{*}$. Let $\delta \stackrel{\text { def }}{=} t_{f}-a_{0}$, the length of the time interval in which there are pending jobs from $S^{*}$.

Facts 3 and 4 yield the following two inequalities:

$$
\begin{cases}C_{1} & \leq \ell_{\mathrm{LO}} \times\left(a_{0}+x \delta\right) \\ C_{1}+C_{2} & \leq \ell_{\mathrm{LO}} \times\left(a_{0} / x+\delta\right) .\end{cases}
$$

By definition of $U_{\mathrm{HI}}^{\mathrm{HI}}$, we also obtain another set of inequalities

$$
\begin{cases}C^{*} & \leq U_{\mathrm{HI}}^{\mathrm{HI}} \delta \\ C^{*}+C_{2} & \leq U_{\mathrm{HI}}^{\mathrm{HI}} \times\left(a_{0} / x+\delta\right) .\end{cases}
$$

Since we have assumed that $J$ misses its deadline $t_{f}=a_{0}+$ $\delta$, it must be the case that $C_{1}+C_{2}+C^{*}>t_{f}$, which is equivalent to $C_{1}+C_{2}+C^{*}-a_{0}>\delta$. Hence the maximum value of $C_{1}+C_{2}+C^{*}-a_{0}$ over all possible $a_{0}$ must be greater than $\delta$. We will now attempt to derive conditions necessary for this to be the case. For notational convenience, let us henceforth refer to $a_{0}$ as $\alpha$, and study the function

$$
o(\alpha) \stackrel{\text { def }}{=} C_{1}+C_{2}+C^{*}-\alpha
$$

From inequalities (8) and (9), we get the following two upper bounds, $f(\alpha)$ and $g(\alpha)$, on the function $o(\alpha)$ :

$$
\begin{aligned}
& f(\alpha) \stackrel{\text { def }}{=} \ell_{\mathrm{LO}} \times(\alpha+x \delta)+U_{\mathrm{HI}}^{\mathrm{HI}} \times(\alpha / x+\delta)-\alpha \\
& g(\alpha) \stackrel{\text { def }}{=} \ell_{\mathrm{LO}} \times\left(\frac{\alpha}{x}+\delta\right)+U_{\mathrm{HI}}^{\mathrm{HI}} \times \delta-\alpha .
\end{aligned}
$$

As observed at the beginning of the proof of Lemma 2, the value assigned to $x$ at Line L2 of our algorithm is $\geq \ell_{\mathrm{LO}}$, which in turn implies that $\ell_{\mathrm{LO}} / x \leq 1$. Using this fact in conjunction with the observation that

$$
\left(\ell_{\mathrm{LO}}+U_{\mathrm{HI}}^{\mathrm{HI}}\right) \geq\left(U_{\mathrm{LO}}^{\mathrm{LO}}+U_{\mathrm{HI}}^{\mathrm{HI}}\right)>1
$$

(since the condition of Line $\mathrm{C} 1$ had evaluated to false), we obtain the following bounds on the derivatives of $f$ and $g$

$$
\begin{aligned}
& \frac{\partial f(\alpha)}{\partial \alpha}=\ell_{\mathrm{LO}}+U_{\mathrm{HI}}^{\mathrm{HI}} / x-1 \geq \ell_{\mathrm{LO}}+U_{\mathrm{HI}}^{\mathrm{HI}}-1>0 \\
& \frac{\partial g(\alpha)}{\partial \alpha}=\ell_{\mathrm{LO}} / x-1 \leq 1-1=0,
\end{aligned}
$$

Hence $f(\alpha)$ increases and $g(\alpha)$ does not increase, with increasing $\alpha$ for $\alpha \in(0,+\infty)$. Thus $\max _{\alpha} o(\alpha)$ is always bounded from above by the values of $f$ and $g$ at the point where the lines $f(\alpha)$ and $g(\alpha)$ intersect. Let $\gamma$ denote the value of $\alpha$ for which this happens: $f(\gamma)=g(\gamma)$. Straightforward algebraic simplification yields

$$
\gamma=\frac{\ell_{\mathrm{LO}} \times(1-x) x \delta}{U_{\mathrm{HI}}^{\mathrm{HI}}-\ell_{\mathrm{LO}} \times(1-x)},
$$

and therefore

$$
\begin{aligned}
\max _{\alpha} o(\alpha) \leq \\
\quad\left(\frac{U_{\mathrm{HI}}^{\mathrm{HI}}+\ell_{\mathrm{LO}} U_{\mathrm{HI}}^{\mathrm{HI}} \times x-\ell_{\mathrm{LO}} \times x+\ell_{\mathrm{LO}} \times x^{2}}{U_{\mathrm{HI}}^{\mathrm{HI}}-\ell_{\mathrm{LO}}+\ell_{\mathrm{LO}} \times x} \delta\right)
\end{aligned}
$$

Hence, if $J$ misses its deadline as we assumed, we must have $o(\gamma)>\delta$; that is,

$$
\ell_{\mathrm{LO}} \times x^{2}-\left(2-U_{\mathrm{HI}}^{\mathrm{HI}}\right) \ell_{\mathrm{LO}} \times x+\ell_{\mathrm{LO}}-U_{\mathrm{HI}}^{\mathrm{HI}}+U_{\mathrm{HI}}^{\mathrm{HI}}>0 .
$$

We have just shown that Inequality 14 represents a necessary condition for a deadline miss. Taking its negation, we conclude

\section{FACT 5. If the following condition is satisfied}

$$
\ell_{\mathrm{LO}} \times x^{2}-\left(2-U_{\mathrm{HI}}^{\mathrm{HI}}\right) \ell_{\mathrm{LO}} \times x+\ell_{\mathrm{LO}}-U_{\mathrm{HI}}^{\mathrm{HI}}+U_{\mathrm{HI}}^{\mathrm{HI}} \leq 0
$$

then no deadline is missed in any $\mathrm{HI}$-criticality behavior.

We now have the pieces in place to prove that our algorithm schedules every HI-criticality behavior correctly, if it declares success at Line L2. 


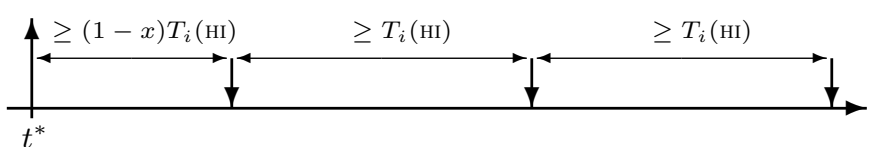

Figure 2: Deadlines of HI-criticality task $\tau_{i}$ after a criticality level change occurs at $t^{*}$. The first deadline is at least $(1-x) T_{i}(\mathrm{HI})$ time away; successive deadlines are at least $T_{i}(\mathrm{HI})$ time apart.

LEMMA 3. If the algorithm returns success at Line L2, then no deadline is missed by the run-time dispatcher described in Section 3.2 upon any HI-criticality behavior.

Proof: For Line L2 of Algorithm 1 to have executed, it must be the case that the condition $\mathrm{C} 2$ evaluates to true; i.e., that

$$
\begin{aligned}
U_{\mathrm{HI}}^{\mathrm{HI}} & \leq \frac{4\left(1-\ell_{\mathrm{LO}}\right)}{4-\ell_{\mathrm{LO}}} \\
& \Leftrightarrow \quad 4 U_{\mathrm{HI}}^{\mathrm{HI}}-U_{\mathrm{HI}}^{\mathrm{HI}} \ell_{\mathrm{LO}} \leq 4-4 \ell_{\mathrm{LO}} \\
& \Leftrightarrow \quad 4 U_{\mathrm{HI}}^{\mathrm{HI}}-U_{\mathrm{HI}}^{\mathrm{HI}} \ell_{\mathrm{LO}}-4+4 \ell_{\mathrm{LO}} \leq 0 \\
& \Leftrightarrow \quad 4\left(U_{\mathrm{HI}}^{\mathrm{HI}}-\frac{U_{\mathrm{HI}}^{\mathrm{HI}} \ell_{\mathrm{LO}}}{4}-1+\ell_{\mathrm{LO}}\right) \leq 0 \\
& \Leftrightarrow \quad U_{\mathrm{HI}}^{\mathrm{HI}}-\frac{U_{\mathrm{HI}}^{\mathrm{HI}} \ell_{\mathrm{LO}}}{4}-1+\ell_{\mathrm{LO}} \leq 0
\end{aligned}
$$

Let us now simplify the LHS of Inequality 15 when $x$ is assigned the value $\left(\frac{2-U_{\mathrm{HI}}^{\mathrm{HI}}}{2}\right)$ :

$$
\begin{aligned}
& \ell_{\mathrm{LO}} \times x^{2}-\left(2-U_{\mathrm{HI}}^{\mathrm{HI}}\right) \ell_{\mathrm{LO}} \times x+\ell_{\mathrm{LO}}-U_{\mathrm{HI}}^{\mathrm{HI}}+U_{\mathrm{HI}}^{\mathrm{HI}} 2 \\
& =\ell_{\mathrm{LO}} \times\left(\frac{2-U_{\mathrm{HI}}^{\mathrm{HI}}}{2}\right)^{2}-\left(2-U_{\mathrm{HI}}^{\mathrm{HI}}\right) \ell_{\mathrm{LO}} \times\left(\frac{2-U_{\mathrm{HI}}^{\mathrm{HI}}}{2}\right) \\
& +\ell_{\mathrm{LO}}-U_{\mathrm{HI}}^{\mathrm{HI}}+U_{\mathrm{HI}}^{\mathrm{HI} 2} \\
& =\frac{\ell_{\mathrm{LO}}}{4}\left(2-U_{\mathrm{HI}}^{\mathrm{HI}}\right)^{2}-\frac{\ell_{\mathrm{LO}}}{2}\left(2-U_{\mathrm{HI}}^{\mathrm{HI}}\right)^{2}+\ell_{\mathrm{LO}}-U_{\mathrm{HI}}^{\mathrm{HI}}+U_{\mathrm{HI}}^{\mathrm{HI}} \\
& =-\frac{\ell_{\mathrm{LO}}}{4}\left(2-U_{\mathrm{HI}}^{\mathrm{HI}}\right)^{2}+\ell_{\mathrm{LO}}-U_{\mathrm{HI}}^{\mathrm{HI}}+U_{\mathrm{HI}}^{\mathrm{HI}} \\
& =-\frac{\ell_{\mathrm{LO}}}{4}\left(4-4 U_{\mathrm{HI}}^{\mathrm{HI}}+U_{\mathrm{HI}}^{\mathrm{HI}}\right)+\ell_{\mathrm{LO}}-U_{\mathrm{HI}}^{\mathrm{HI}}+U_{\mathrm{HI}}^{\mathrm{HI}} \\
& =-\ell_{\mathrm{LO}}+\ell_{\mathrm{LO}} U_{\mathrm{HI}}^{\mathrm{HI}}-\frac{\ell_{\mathrm{LO}} U_{\mathrm{HI}}^{\mathrm{HI}} 2}{4}+\ell_{\mathrm{LO}}-U_{\mathrm{HI}}^{\mathrm{HI}}+U_{\mathrm{HI}}^{\mathrm{HI}} 2 \\
& =U_{\mathrm{HI}}^{\mathrm{HI}}\left(\ell_{\mathrm{LO}}-\frac{\ell_{\mathrm{LO}} U_{\mathrm{HI}}^{\mathrm{HI}}}{4}-1+U_{\mathrm{HI}}^{\mathrm{HI}}\right) \\
& \leq 0 \text { (By Inequality } 16 \text { above) }
\end{aligned}
$$

and hence by Fact 5, no HI-criticality deadlines are missed.

\subsection{Returns success at Line L4}

We now turn our attention to the third and last point at which Algorithm 1 may declare success. This case - the pseudo-code between the lines labeled L3 and L5 in Algorithm 1 - essentially emulates the algorithm that was derived and proved correct in [1]; its correctness therefore follows from the proofs in [1]. We outline the essence of those proofs in the proof of Lemma 4 below.

LEMMA 4. If the algorithm returns success at Line L4 for a given task system $\tau$, then $\tau$ is scheduled correctly by the run-time dispatcher described in Section 3.2.
Proof: In Line L3, we seek the smallest value of $x$ such that the (regular) sporadic task system $\hat{\tau}(x)$ is EDF-schedulable. It is straightforward to show, using techniques analogous to those used in the proof of Lemma 2 , that $\hat{\tau}\left(\ell_{\mathrm{LO}}\right)$ is indeed EDF-schedulable; hence the value of $x$ we seek lies in the range $\left[0, \ell_{\mathrm{LO}}\right]$. It can be obtained to any desired degree of accuracy by bisection search ("binary search") for the smallest value of $x$ such that task system $\hat{\tau}(x)$ is deemed EDFschedulable by one of the schedulability tests described in $[6$, $13,14]$.

It is evident that for this value of $x$, no deadline is missed by the run-time dispatcher described in Section 3.2 upon any LO-criticality behavior. It remains to determine whether any deadlines may be missed in any HI-criticality behavior. To check this, we test (line C3) whether the regular 3-parameter sporadic task system,

$$
\bigcup_{\chi_{i}=\mathrm{HI}}\left\{\hat{\tau}_{i}(1-x)\right\}
$$

is EDF-schedulable or not. If so, $\tau$ passes our schedulability test; otherwise, we declare failure and return. Figure 2 briefly illustrates why testing the EDF-schedulability of the 3-parameter sporadic task system depicted in Equation 17 above is sufficient for ensuring that no deadline is missed by the run-time dispatcher described in Section 3.2 upon any HI-criticality behavior. It is evident from inspection of this figure that the demand bound function for a HI-criticality task $\tau_{i}$ after the instant $t^{*}$ at which the criticality level change occurs is exactly equal to the demand bound function of a 3-parameter sporadic task with WCET $C_{i}$, relative deadline $(1-x) T_{i}(\mathrm{HI})$, and period $T_{i}$. Hence, validating that the 3 -parameter sporadic task system depicted in Equation 17 above is EDF-schedulable is sufficient for ensuring that no deadlines are missed by our EDF-based run-time dispatcher in any HI-criticality behavior.

\section{PROPERTIES}

In Section 4 above, we proved that Algorithm 1 of Section 3.1 , when used in conjunction with the run-time dispatcher of Section 3.2, represents a correct scheduling strategy for MC LL task systems in which multiple period parameters are specified for each task. In this section, we provide an evaluation of our scheduling algorithm.

\subsection{Run-time complexity}

The preprocessing step (Algorithm 1) starts out requiring that $U_{\mathrm{LO}}^{\mathrm{LO}}$ and $U_{\mathrm{HI}}^{\mathrm{HI}}$ be computed - this can be done in time linear in the number of tasks in the system. The quantity $\ell_{\mathrm{LO}}$ may additionally need to be computed; for task systems for which LO-criticality utilization is bounded from above by a constant strictly less than one, this can be done in time pseudo-polynomial in the representation of the task system $[6,13,14]$, or approximately to any desired degree of accuracy in polynomial time [9] — see the discussion in Section 2.1. If Algorithm 1 fails to return at Lines L1 or $\mathrm{L} 2$, then an iterative process of computing the scaling factor $x$, and of validating this $x$, would require multiple calls to a pseudo-polynomial time exact or polynomial-time approximate algorithm, the number of calls being determined by the desired degree of accuracy.

In Sections 5.3 and 5.4 below, we will provide quantitative characterizations of the efficacy of this algorithm. These characterizations continue to hold even if the preprocessing 


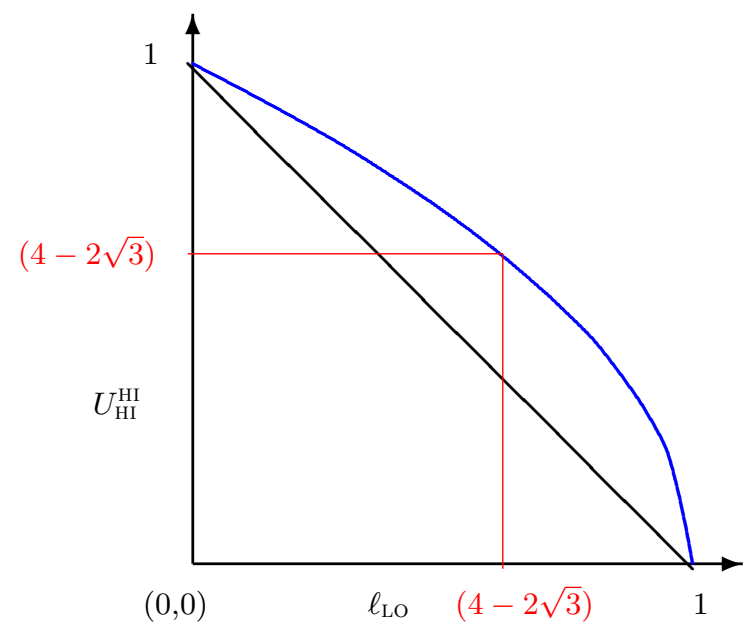

Figure 3: Feasibility region for Algorithm 1. The $x$ axis denotes the LO-criticality load $\ell_{\mathrm{LO}}$; the $y$ axis, the HI-criticality utilization $U_{\mathrm{HI}}^{\mathrm{HI}}$. The curved line satisfies $U_{\mathrm{HI}}^{\mathrm{HI}}=4\left(1-\ell_{\mathrm{LO}}\right) /\left(4-\ell_{\mathrm{LO}}\right)$. Any instance with $\ell_{\mathrm{LO}}$ and $U_{\mathrm{HI}}^{\mathrm{HI}}$ bounded between this line and the axes is guaranteed schedulable by Algorithm 1.

algorithm (Algorithm 1) simply declares failure upon failing to return at Lines L1 or L2; hence, it is safe to declare that the algorithm being evaluated quantitatively in Sections 5.35.4 has pseudo-polynomial run-time complexity.

The run-time dispatcher of Section 3.2 is essentially implementing preemptive uniprocessor EDF, for which very efficient implementations are known that have logarithmic run-time complexity per scheduling decision (see, e.g., [12]). A naive implementation of the run-time dispatcher could result in linear time-complexity if the criticality level-change occurs; however, efficient implementations of this operation are known as well $[2,3]$ that implement such a change in logarithmic time.

\subsection{Dominance over the algorithm in [1]}

Prior to the work reported in this document, the state of the art for scheduling MC LL task systems with multiple periods specified for the tasks was the algorithm in [1]. Algorithm 1 strictly generalizes this algorithm by including it as a "catch-all" in lines L2-L5; hence Algorithm 1 will trivially successfully schedule any instance that was successfully scheduled by the algorithm of [1].

\subsection{Feasibility region}

It is evident that any instance $\tau$ with $\ell_{\mathrm{LO}}>1$ or $U_{\mathrm{HI}}^{\mathrm{HI}}>1$ cannot possibly be scheduled on a unit-speed processor to always meet all deadlines; hence,

$$
\left(\ell_{\mathrm{LO}} \leq 1 \wedge U_{\mathrm{HI}}^{\mathrm{HI}} \leq 1\right)
$$

is a necessary condition for schedulability. If

$$
U_{\mathrm{HI}}^{\mathrm{HI}} \leq \frac{4\left(1-\ell_{\mathrm{LO}}\right)}{4-\ell_{\mathrm{LO}}},
$$

then Algorithm 1 will deem the instance schedulable (and return success in Line L2). The curve $U_{\mathrm{HI}}^{\mathrm{HI}}=4\left(1-\ell_{\mathrm{LO}}\right) /(4-$ $\left.\ell_{\mathrm{LO}}\right)$ is depicted in Figure 3 ; any instance $\tau$ for which $\ell_{\mathrm{LO}}$ and $U_{\mathrm{HI}}^{\mathrm{HI}}$ falls below this curve is successfully scheduled by
Algorithm 1 in conjunction with the run-time dispatcher of Section 3.2 .

\subsection{Speedup}

A scheduling algorithm $A$ is said to have a speedup bound $b$, where $b$ is a positive real number $\geq 1$, if any task system schedulable by an optimal clairvoyant scheduler upon a unitspeed processor is also schedulable by algorithm $A$ upon a speed- $b$ processor. Speedup factor is thus one metric of effectiveness for scheduling algorithms - the smaller the speedup factor, the better the algorithm (with optimal algorithms having speedup factor 1 ).

As stated in Section 5.3 above, any instance with $U_{\mathrm{HI}}^{\mathrm{HI}} \leq$ $4\left(1-\ell_{\mathrm{LO}}\right) /\left(4-\ell_{\mathrm{LO}}\right)$ is guaranteed schedulable by Algorithm 1 . If we choose $\ell_{\mathrm{LO}} \leftarrow(4-2 \sqrt{3})$, we have that the corresponding upper bound in $U_{\mathrm{HI}}^{\mathrm{HI}}$ is

$$
\begin{aligned}
& \frac{4(1-(4-2 \sqrt{3}))}{4-(4-2 \sqrt{3})} \\
= & \frac{4(-3+2 \sqrt{3})}{2 \sqrt{3}} \\
= & \frac{4 \sqrt{3}(2-\sqrt{3})}{2 \sqrt{3}} \\
= & 2(2-\sqrt{3}) \\
= & (4-2 \sqrt{3})
\end{aligned}
$$

Observing that $\frac{1}{(4-2 \sqrt{3})}=1+\frac{\sqrt{3}}{2}$,

we conclude that

LEMma 5. Any instance that is schedulable correctly upon a particular preemptive uniprocessor platform by an optimal clairvoyant scheduler is scheduled correctly by Algorithm 1 upon a preemptive uniprocessor platform that is $\left(1+\frac{\sqrt{3}}{2}\right)$ times as fast.

That is, Algorithm 1 has a speedup bound $\left(1+\frac{\sqrt{3}}{2}\right)$.

\section{CONCLUSIONS}

Mixed-criticality scheduling has recently emerged as an exciting and important area of research in the real-time systems community. Most of the prior research on mixedcriticality scheduling has focused upon dealing with uncertainty in WCET estimates. However, epistemic uncertainty regarding external events that trigger a system response is an additional important form of uncertainty in many safetycritical systems; in this work we have attempted to understand how to deal with such uncertainty when it is characterized by multiple estimates, at differing levels of assurance, of the minimum inter-arrival separation between successive triggering events. We have derived, and proved the correctness of, an algorithm for scheduling collections of such tasks upon preemptive uniprocessor platforms. We have also provided quantitative characterizations of the efficacy of our algorithm; to our knowledge, this is the first quantitatively characterized algorithm for scheduling mixed-criticality systems with multiple frequency specifications.

In this paper, we have focused solely upon multiple frequency estimations, completely decoupling this from the issue of multiple WCET estimations. In fact our results generalize to concurrent consideration of both sources of uncertainty — i.e., a model in which multiple estimations of both 
the frequency and the WCET are provided; however, the presentation of this generalized algorithm (and particularly its proof) is considerably more messy than the presentation here, and so for pedantic reasons we have chosen to only discuss the frequency dimension.

It would be interesting and important to extend our techniques and results to systems with more than two criticality levels specified. Although we know how to generalize the algorithm to this manner, we have not been able to obtain closed-form speedup bounds for the generalized algorithm indeed, obtaining such closed-form speedup bounds remains open even for the simpler, and far more widely studied, problem in which multiple specifications are provided only along the WCET problem. We leave this as future work.

\section{Acknowledgements}

This research has been supported in part by NSF grants CNS 1115284, CNS 1218693, CNS 1409175, and CPS 1446631, AFOSR grant FA9550-14-1-0161, ARO grant W911NF-141-0499, and a grant from General Motors Corp.

\section{REFERENCES}

[1] S. Baruah. Certification-cognizant scheduling of tasks with pessimistic frequency specification. In Proceedings of the IEEE Symposium on Industrial Embedded Systems (SIES). IEEE Press, June 2012.

[2] S. Baruah, V. Bonifaci, G. D'Angelo, H. Li, A. Marchetti-Spaccamela, S. van der Ster, and L. Stougie. The preemptive uniprocessor scheduling of mixed-criticality implicit-deadline sporadic task systems. In Proceedings of the 2012 24th Euromicro Conference on Real-Time Systems, ECRTS '12, Pisa (Italy), 2012. IEEE Computer Society.

[3] S. Baruah, V. Bonifaci, G. D'Angelo, H. Li, A. Marchetti-Spaccamela, S. van der Ster, and L. Stougie. Preemptive uniprocessor scheduling of mixed-criticality sporadic task systems. Journal of the ACM, 62(2), 2015.

[4] S. Baruah and A. Burns. Implementing mixed criticality systems in Ada. In A. Romanovsky and T. Vardanega, editors, Proceedings of Reliable Software Technology - Ada Europe 2011, volume LNCS 6652, pages 174-188. Springer, 2011.

[5] S. Baruah and B. Chattopadhyay. Response-time analysis of mixed criticality systems with pessimistic frequency specification. In Proceedings of the IEEE International Conference on Embedded and Real-Time Computing Systems and Applications (RTCSA), 2013.

[6] S. Baruah, A. Mok, and L. Rosier. Preemptively scheduling hard-real-time sporadic tasks on one processor. In Proceedings of the 11th Real-Time Systems Symposium, pages 182-190, Orlando, Florida, 1990. IEEE Computer Society Press.

[7] A. Burns and R. Davis. Mixed criticality on controller area network. In Proceedings of the 2013 25th Euromicro Conference on Real-Time Systems, ECRTS '13, pages 125-134, Paris (France), 2013.

[8] A. Burns and R. Davis. Mixed-criticality systems: A review (6th edition). Available at http://wwwusers.cs.york.ac.uk/ burns/review.pdf, 2015.

[9] N. Fisher, T. Baker, and S. Baruah. Algorithms for determining the demand-based load of a sporadic task system. In Proceedings of the International Conference on Real-time Computing Systems and Applications, Sydney, Australia, August 2006. IEEE Computer Society Press.

[10] C. Liu and J. Layland. Scheduling algorithms for multiprogramming in a hard real-time environment. Journal of the ACM, 20(1):46-61, 1973.

[11] A. Mok. Fundamental Design Problems of Distributed Systems for The Hard-Real-Time Environment. PhD thesis, Laboratory for Computer Science, Massachusetts Institute of Technology, 1983. Available as Technical Report No. MIT/LCS/TR-297.

[12] A. Mok. Task management techniques for enforcing ED scheduling on a periodic task set. In Proceedings of the 5th IEEE Workshop on Real-Time Software and Operating Systems, pages 42-46, Washington D.C., May 1988.

[13] I. Ripoll, A. Crespo, and A. K. Mok. Improvement in feasibility testing for real-time tasks. Real-Time Systems: The International Journal of Time-Critical Computing, 11:19-39, 1996.

[14] F. Zhang and A. Burns. Schedulability analysis for real-time systems with EDF scheduling. IEEE

Transactions on Computers, 2009. 